\title{
Biorthogonal PPM for Transmitted Reference IR-UWB Communication Systems
}

\author{
Mario VRANJEŠ, Denis VRANJEŠ, Tomislav MATIĆ
}

\begin{abstract}
Research of impulse radio ultra-wideband (IR-UWB) systems has been extensively increasing in recent years due to their high power efficiency, high data rates and low hardware complexity. The choice of the modulation scheme used in an IR-UWB system has a great impact on its overall performances. In this paper, the biorthogonal pulse position modulation (BPPM) scheme for the application in transmitted reference (TR) IR-UWB communication systems is proposed. Performances of the BPPM scheme over two different IEEE 805.15.3a channel models in a multi-user environment are analysed and compared to the performances of the pulse position modulation (PPM) scheme. The results show that the BPPM scheme outperforms the PPM scheme in terms of bit error rate (BER), data rate and hardware complexity. Additionally, the influence of different system parameters on the BPPM performances in TR IR-UWB systems is analysed and possible trade-offs are proposed.
\end{abstract}

Keywords: bit error rate; multi-user interference; pulse position modulation; ultra-wideband

\section{INTRODUCTION}

Trends in modern communication systems place high demands on low power consumption, low hardware complexity, high data rate and high anti-interference characteristics. Therefore, impulse radio ultra-wideband (IR-UWB) systems have recently gained increased popularity. Since the information in IR-UWB systems is transmitted with short pulses $(<2 \mathrm{~ns})$, signal energy has been spread over the frequency band of up to $10 \mathrm{GHz}$ [1] Many modulation schemes for the application in IR-UWB systems are proposed, like pulse amplitude modulation (PAM) [2], pulse position modulation (PPM) $[3,4]$, pulse shape modulation (PSM) [5], on-off-keying (OOK) [6], binary phase-shift keying (BPSK) $[7,8]$, pulse interval modulation (PIM) [9], etc.

Due to the large number of multipath components in IR-UWB channels [10], in coherent IR-UWB systems, a complex RAKE receiver is required for collecting of signal energy for proper demodulation. In IR-UWB systems the pulses are distorted during the transmission over the channel and at the receiver the template signal used for the proper detection should have the same shape as the distorted received signal [11], so accurate channel estimation should be performed. Due to this channel estimation process, the usage of the RAKE receiver significantly increases the hardware complexity. This led to the proposition of the transmitted reference (TR) IRUWB systems [12].

In TR IR-UWB systems the symbol frame consists of two pulses - the reference one followed by the data one. Both of them experience the same channel conditions. Thus the delayed reference pulse is used as the template signal for the proper detection of appropriate information contained in data pulse. So, there is no need for channel estimation at the receiver. In TR systems the correlation between received data pulse and delayed received reference pulse is calculated over a proper time interval. Due to the large number of multipath components [10], the integration time interval is a significant parameter since it determines the amount of collected signal energy contained in different multipath components which arrive to the receiver. In TR IR-UWB symbol frame, the separation time, $T_{d}$, between the reference pulse and the data pulse should be less than the channel coherence time, in order that both pulses experience the same channel conditions. On the other hand, to avoid inter-pulse interference (IPI), $T_{d}$ should be equal or larger than channel delay spread, $T_{m d s}$. This is a disadvantage of TR IR-UWB systems because this requirement reduces the data rate. However, in $[13,14]$ authors presented different methods to resolve this problem and to increase the data rate of such systems.

Most of the current researches of TR IR-UWB systems are based on binary modulation schemes like BPSK [15] and binary PPM [16]. To increase the bandwidth efficiency of such systems, authors presented $M$-ary PPM in [17]. In this paper, $M$-ary biorthogonal pulse position modulation (BPPM) for the TR IR-UWB systems is proposed. Although the BPPM scheme for ordinary coherent IR-UWB systems was presented in [18], the BPPM scheme for the application in TR IRUWB systems has not been analyzed yet. In this paper we performed this analysis and showed that the proposed BPPM scheme has better performances (lower bit error rate (BER) and higher data rate) and lower hardware complexity than the $M$-ary PPM scheme when using in TR IR-UWB systems.

The paper is organized as follows. In Section 2 the TR IR-UWB BPPM system model is described, while in Section 3 the system performance analysis is made. Numerical results are presented in Section 4, whereas concluding remarks are given in Section 5.

\section{TR IR-UWB BPPM SYSTEM MODEL 2.1 Transmitted Signal}

In the $M$-ary BPPM scheme a signal set is formed using $2^{k}$ orthogonal PPM signals and their respective antipodal versions, which then form an overall set of $M=2^{k+1}$ possible signals. Parameter $k$ represents the number of bits per symbol in the PPM scheme. If the multi-user system is considered, the $v^{\text {th }}$ user transmitted $M$-ary BPPM TR IR-UWB signal is given by

$$
\begin{aligned}
& s^{(v)}(t)=\sum_{v=1}^{N_{u}} \sum_{j=-\infty}^{\infty} \sqrt{E_{p}}\left[p^{(v)}\left(t-j T_{f}-c_{j}^{(v)} T_{c}\right)+\right. \\
& \left.+b_{\left\lfloor j / N_{S}\right\rfloor}^{(v)} p^{(v)}\left(t-j T_{f}-c_{j}^{(v)} T_{c}-T_{d}^{(v)}-d_{\left\lfloor j / N_{S}\right\rfloor}^{(v)} \Lambda\right)\right]
\end{aligned}
$$


where $j$ is a frame index, $E_{p}$ is symbol energy, $N_{u}$ is the number of users, $p^{(v)}(t)$ is the $v^{\text {th }}$ user pulse waveform with pulse width $T_{p}$ and unit energy $\int_{-\infty}^{\infty}\left(p^{(v)}\right)^{2}(t) \mathrm{d} t=1 . T_{f}$ is a frame duration which determines the symbol duration as $T_{S}=N_{S} T_{f}$, where $N_{S}$ is the number of frames used to transmit a single symbol. $c_{j}^{(v)}$ is the $v^{\text {th }}$ user pulse shift pattern, also called time hopping $(\mathrm{TH})$ sequence, which is a pseudorandom variable and can take any integer value from the range $\left[0, N_{h}^{(v)}-1\right]$. The separation time between the reference pulse and the data pulse for the $v^{\text {th }}$ user in multi-user environment is defined as

$$
T_{d}^{(v)}=T_{m d s}+(v-1) T_{p},
$$

while the number of chips within one frame for the $v^{\text {th }}$ user ( $v^{\text {th }}$ user's maximum TH value) is defined as

$N_{h}^{(v)}=N_{h}^{\left(N_{u}\right)}+\left(N_{u}-v\right)$

$d_{\left\lfloor j / N_{S}\right\rfloor}^{(v)} \in\{0, \ldots, M / 2-1\} \quad$ and $\quad b_{\left\lfloor j / N_{S}\right\rfloor}^{(v)} \in\{-1,1\} \quad$ are determined with the information sent by the $v^{\text {th }}$ source and represent the position and amplitude level of data pulse in $M$-ary BPPM symbol, respectively. $T_{c}$ is the chip duration time and it is equal to $T_{p}$, while $\Lambda$ is a time shift parameter and it should be at least equal to $T_{m d s}$ in order to avoid IPI.

\subsection{UWB Channel Model}

In this work a discrete channel model proposed in [19] is used, in which the time axis is divided into discrete time bins with duration $\Delta=0,167 \mathrm{~ns}$. In this model in any time bin, one or more multipath components can arrive due to the cluster overlapping. Then the channel gain coefficients of all multipath components arrived within one time bin are summed to yield an overall channel gain coefficient for that bin. The channel impulse response for the $v$-th user can be represented as

$h^{(v)}(t)=\sum_{l=1}^{L_{t}} \alpha_{l}^{(v)} \delta(t-l \Delta)$,

where $L_{t}$ is the total number of available multipath components and $\alpha_{l}^{(v)}$ represents the sum of channel gain coefficients of all multipath components arrived in $l^{\text {th }}$ time bin. $\delta(t)$ is the Dirac delta function, while $\Delta$ is chosen to be much less than the channel delay spread, so that the $\left\{\alpha_{l}^{(v)}\right\}_{l=1}^{L_{t}}$ related to different time bins can be considered to be uncorrelated. In order to avoid the pulse distortion due to the partial pulse overlapping, the pulse width $T_{p}$ is set to $0,167 \mathrm{~ns}$, as in $[13,19,20]$.

\subsection{TR receiver}

Without loss of generality, it can be assumed that the first user is desired user, while the signals of all other users are assumed to cause the interference at the receiver (multi-user interference (MUI)). After transmission through the channel, the received signal that corresponds to the $z^{\text {th }}$ symbol of the first user can be written as $r(t)=s(t)+i(t)+n(t)$, where

$$
\begin{aligned}
& s(t)=\sum_{j=z N_{s}}^{(z+1) N_{s}-1} \sqrt{E_{p}}\left[g^{(1)}\left(t-j T_{f}-c_{j}^{(1)} T_{c}-\tau_{1}\right)+\right. \\
& \left.+b_{z}^{(1)} g^{(1)}\left(t-j T_{f}-c_{j}^{(1)} T_{c}-T_{d}^{(1)}-d_{z}^{(1)} \Lambda-\tau_{1}\right)\right] \\
& i(t)=\sum_{v=2}^{N_{u}} \sum_{j=-\infty}^{\infty} \sqrt{E_{p}}\left[g^{(v)}\left(t-j T_{f}-c_{j}^{(v)} T_{c}-\tau_{v}\right)+\right. \\
& \left.+b_{\left\lfloor j / N_{s}\right\rfloor}^{(v)} g^{(v)}\left(t-j T_{f}-c_{j}^{(v)} T_{c}-T_{d}^{(v)}-d_{\left\lfloor j / N_{s}\right\rfloor}^{(v)} \Lambda-\tau_{v}\right)\right]
\end{aligned},
$$

while $n(t)$ is the filtered additive white Gaussian noise (AWGN) with power spectral density of $N_{0} / 2 . \tau_{v}$ is the time offset between the first user and the $v$-th user (the time asynchronism between the clocks of transmitter $v$ and the receiver), while $g^{(v)}(t)=p^{(v)}(t) \otimes h^{(v)}(t)$ is the received pulse waveform of the $v^{\text {th }}$ user, where $\otimes$ denotes convolution.

Assuming perfect synchronization between transmitter and receiver of the first user, i.e. $\tau_{1}=0$, the time offset $\tau_{v}(v \neq 1)$ can be modeled as a random variable uniformly distributed over the interval $\left[0, T_{f}\right]$, [15]. In order to avoid inter-frame interference, all possible symbols have to be comprised within one frame and thus the frame time $T_{f}$ has to be large enough. Therefore the similar assumption, but approximately double decreased, as in [17] is adopted:

$$
T_{f} \geq \max \left\{N_{h}^{(v)} T_{c}+T_{d}^{(v)}+M \Lambda\right\}
$$

TR IR-UWB BPPM receiver is shown in Fig. 1. For all possible modulation slots, the correlation between the reference signal and the signal of corresponding modulation slot is calculated, producing $M / 2$ correlation outputs $\left(C_{0, z}, \ldots C_{M / 2-1, z}\right)$. After that, based on the largest absolute value of the correlation outputs and the sign of this value, the detector decides which combination of possible positions and amplitudes has been transmitted.

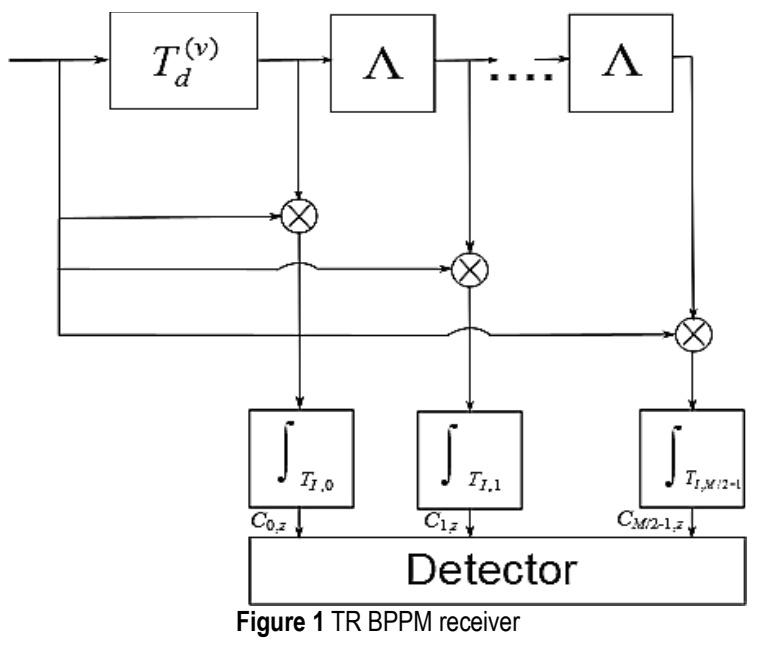

For the first user, the correlation output for the $z^{\text {th }}$ symbol in $m^{\text {th }}$ slot can be written as: 


$$
C_{m, z}=\sum_{j=z N_{S}}^{(z+1) N_{S}-1} \int_{T_{I, m}} r(t) r\left(t-T_{d}^{(1)}-m \Lambda\right) \mathrm{d} t,
$$

where $m$ is an integer value, $0 \leq m \leq M / 2-1$, and

$$
\begin{aligned}
& T_{I, m}=\left[j T_{f}+T_{d}^{(1)}+c_{j}^{(1)} T_{c}+m \Lambda,\right. \\
& \left.j T_{f}+T_{d}^{(1)}+c_{j}^{(1)} T_{c}+m \Lambda+T_{\text {corr }}\right]
\end{aligned}
$$

is the integration time interval of the $m^{\text {th }}$ slot. $T_{\text {corr }}$ is assumed to be an integer multiple of the pulse width, denoted by $L_{p} T_{p}$, where $L_{p} \in\left\{1,2, \ldots L_{t}\right\}$.

\section{PERFORMANCE ANALYSIS}

Without loss of generality, it can be assumed that BPPM symbol with data pulse positioned in the first modulation slot $\left(d_{z}^{(1)}=0\right)$ with amplitude value $1\left(b_{z}^{(1)}=1\right)$ is sent. Correlation outputs for the given conditions are

$$
C_{m, z}= \begin{cases}\varepsilon_{p}+N_{m, 1}+N_{m, 2}+I_{m, 1}+I_{m, 2}+I_{m, 3}, & \text { for } m=0 \\ N_{m, 1}+N_{m, 2}+I_{m, 1}+I_{m, 2}+I_{m, 3}, & \text { for } m \neq 0\end{cases}
$$

where $\varepsilon_{p}$ is the desired signal. $N_{m, 1}, N_{m, 2}, I_{m, 1}, I_{m, 2}$ and $I_{m, 3}$ are signal-times-noise, noise-times-noise, MUI-timessignal, MUI-times-noise and MUI-times-MUI term, respectively. In this work an assumption from [15, 17] is adopted, where all of them are modeled as zero mean Gaussian random variables.

Using the similar notation as in [14], desired signal for the $z^{\text {th }}$ symbol can be written as

$$
\begin{aligned}
& \varepsilon_{p}=\int_{T_{I, m 1}} s(t) s\left(t-T_{d}^{(1)}-m \Lambda\right) \mathrm{d} t= \\
& =\sum_{j=z N_{S}}^{(z+1) N_{s}-1} E_{p}\left[\int_{T_{d}^{(1)}+m \Lambda}^{T_{d}^{(1)}+m \Lambda+T_{\text {corr }}} g^{(1)}(t) g^{(1)}\left(t-T_{d}^{(1)}-m \Lambda\right) \mathrm{d} t+\right. \\
& +\int_{m}^{T_{d}^{(1)}+m \Lambda+T_{\text {corr }}} g^{(1)}(t) g^{(1)}\left(t-2 T_{d}^{(1)}-m \Lambda\right) \mathrm{d} t+ \\
& +\int_{m \Lambda}^{T_{d}^{(1)}+m \Lambda} g^{(1)}(t) g^{(1)}(t-m \Lambda) \mathrm{d} t+ \\
& \left.+\int_{m \Lambda+T_{\text {corr }}}^{m} g^{(1)}(t) g^{(1)}\left(t-T_{d}^{(1)}-m \Lambda\right) \mathrm{d} t\right] .
\end{aligned}
$$

Note that $\varepsilon_{p}$ is

$$
\varepsilon_{p}=\left\{\begin{array}{cl}
\sum_{j=z N_{S}}^{(z+1) N_{S}-1} E_{p} \int_{0}^{T_{\text {corr }}}\left(g^{(1)}(t)\right)^{2} \mathrm{~d} t, & \text { for } m=0 \\
0, & \text { else. }
\end{array}\right.
$$

The mean value of desired signal is

$$
E\left(\varepsilon_{p}\right)=N_{s} E_{p} \sum_{l=1}^{L_{p}} E\left(\alpha_{l}^{(1)}\right)^{2} \int_{0}^{T_{p}}\left(p^{(1)}(t)\right)^{2} \mathrm{~d} t
$$

where $E\left(\alpha_{l}^{(1)}\right)^{2}$ is the mean value of energy of the $l^{\text {th }}$ multipath component given by [19, Eq. (17)], and $E(*)$ is the mean value operator. $N_{m, 1}$ can be written as

$$
N_{m, 1}=\int_{T_{I, m}}\left[n\left(t-T_{d}^{(1)}-m \Lambda\right) s(t)+n(t) s\left(t-T_{d}^{(1)}-m \Lambda\right)\right] \mathrm{d} t .
$$

By combining (14) and (5), $N_{m, 1}$ can be rewritten as $N_{m, 1}=\sum_{j=z N_{S}}^{(z+1) N_{s}=1}\left[N_{m, 1, j, A}+N_{m, 1, j, B}\right]$, where

$$
\begin{aligned}
& N_{m, 1, j, A}=\int_{T_{I, m}} n\left(t-T_{d}^{(1)}-m \Lambda\right) \sqrt{E_{p}}\left[g ^ { ( 1 ) } \left(t-j T_{f}-c_{j}^{(1)} T_{c}\right.\right. \\
& \left.-\tau_{1}\right)+b_{z}^{(1)} g^{(1)}\left(t-j T_{f}-c_{j}^{(1)} T_{c}-T_{d}^{(1)}-d_{z}^{(1)} \Lambda-\tau_{1}\right] \mathrm{d} t \\
& N_{m, 1, j, B}=\int_{T_{I, m}} n(t) \sqrt{E_{p}}\left[g^{(1)}\left(t-j T_{f}-c_{j}^{(1)} T_{c}-\tau_{1}\right)+\right. \\
& +b_{z}^{(1)} g^{(1)}\left(t-j T_{f}-c_{j}^{(1)} T_{c}-2 T_{d}^{(1)}-d_{z}^{(1)} \Lambda-\tau_{1}-m \Lambda\right] \mathrm{d} t
\end{aligned}
$$

Due to the fact that $N_{m, 1}$ is zero mean random variable, the second order moment of $N_{m, 1, j, A}$ is given by

$$
\begin{aligned}
& E\left(N_{m, 1, j, A}^{2}\right)=\frac{N_{0} E_{p}}{2} E\left[\int_{T_{d}^{(1)}+m \Lambda}^{T_{d}^{(1)}+m \Lambda+T_{\text {corr }}}\left(g^{(1)}(t)\right)^{2} \mathrm{~d} t+\right. \\
& \left.+\int_{m \Lambda}^{m \Lambda+T_{\text {corr }}}\left(g^{(1)}(t)\right)^{2} \mathrm{~d} t+2 \int_{T_{d}^{(1)}+m \Lambda}^{T_{d}^{(1)}+m \Lambda+T_{\text {corr }}} g^{(1)}(t) g^{(1)}\left(t-T_{d}^{(1)}\right) \mathrm{d} t\right]
\end{aligned}
$$

The first and the third integral in (17) always take the value zero because $T_{d}^{(1)} \geq T_{m d s}$. Note that $E\left(N_{m, 1, j, A}^{2}\right)$ will take a nonzero value only for $m=0$. In this case, the second integral from (17) takes a nonzero value due to the fact that the desired signal will be within the integration time interval, while for $m \neq 0$ the integration time interval will not comprise the desired signal. In a similar way the second order moment of $N_{m, 1, j, B}$ can be expressed as

$$
\begin{aligned}
& E\left(N_{m, 1, j, B}^{2}\right)=\frac{N_{0} E_{p}}{2} E\left[\int_{0}^{T_{\text {corr }}}\left(g^{(1)}(t)\right)^{2} \mathrm{~d} t+\right. \\
& \left.+\int_{0}^{T_{\text {corr }}}\left(g^{(1)}\left(t-T_{d}^{(1)}\right)\right)^{2} \mathrm{~d} t+2 \int_{0}^{T_{\text {corr }}} g^{(1)}(t) g^{(1)}\left(t-T_{d}^{(1)}\right) \mathrm{d} t\right]
\end{aligned}
$$

In (18), the first integral takes a nonzero value for all values of $m$, while the second integral will be zero when $T_{c o r r}<T_{d}^{(1)}$. It is also easy to show that the crosscorrelation term $E\left(N_{m, 1, j, A} N_{m, 1, j, B}\right)$ is zero for 
$T_{\text {corr }}<T_{d}^{(1)}$. From (17) and (18) the variance $\operatorname{var}\left(N_{m, 1}\right)=E\left(N_{m, 1, j, A}^{2}\right)+E\left(N_{m, 1, j, B}^{2}\right)$ can be derived as $\operatorname{var}\left(N_{m, 1}\right)=\left\{\begin{array}{l}N_{s} N_{p} N_{0} \sum_{l=1}^{L_{p}} E\left(\left(\alpha_{l}^{(1)}\right)^{2} \int_{0}^{T_{p}}\left(p^{(1)}(t)\right)^{2} \mathrm{~d} t, \text { for } m=0\right. \\ N_{s} N_{p} \frac{N_{0}}{2} \sum_{l=1}^{L_{p}} E\left(\left(\alpha_{l}^{(1)}\right)^{2} \int_{0}^{T_{p}}\left(p^{(1)}(t)\right)^{2} \mathrm{~d} t, \quad \text { else. }\right.\end{array}\right.$

Noise-times-noise term, $N_{m, 2}$, is assumed to be a Gaussian distributed random variable with zero mean and variance as follows [19]:

$\operatorname{var}\left(N_{m, 2}\right)=\frac{N_{s} N_{0}^{2} W T_{c o r r}}{2}$, for all $m$

where $W$ is a receiver front-end filter bandwidth. Before evaluation of the second order moments of MUI terms, the properties of the pulse crosscorrelation function are analyzed. The pulse duration is chosen to be $T_{p}=0,167 \mathrm{~ns}$ in order to avoid IPI caused by multipath components' delays. In that case the crosscorrelation function defined as $\int_{-\infty}^{\infty} p(t) p(t+m \Delta-n \Delta)$ will be equal to 1 only when $\mid m-$ $n \mid=0$, while in all other cases it will be zero. MUItimes-signal term, $I_{m, 1}$, will occur when either the reference or the data pulse of an undesired user falls into integration time interval $T_{I, m}$ of the desired first user, and it can be written as

$$
\begin{aligned}
& I_{m, 1}=\int_{T_{I, m}}\left[s(t) i\left(t-T_{d}^{(1)}-m \Lambda\right)+s\left(t-T_{d}^{(1)}-m \Lambda\right) i(t)\right] \mathrm{d} t= \\
& =\sum_{j=z N_{S}}^{(z+1) N_{s}-1}\left[I_{m, 1, j, A}+I_{m, 1, j, B}\right]
\end{aligned}
$$

Due to the assumption that $\tau_{v}$ is modeled as a random variable, uniformly distributed over interval $\left[0, T_{f}\right]$, it can be easily found that the current and the previous frame of an undesired user can affect the $j^{\text {th }}$ frame of the desired user. Then $I_{m, 1, j, A}$ can be written as

$$
\begin{aligned}
& I_{m, 1, j, A}=\sum_{v=2}^{N_{u}} E_{p} \int_{T_{d}^{(1)}+m \Lambda}^{T_{d}^{(1)}+m \Lambda+T_{c o r r}}\left[\left(g^{(1)}(t)+g^{(1)}\left(t-T_{d}^{(1)}\right)\right) \times\right. \\
& \times\left(g^{(v)}\left(t-\lambda_{j, 0}-T_{d}^{(1)}-m \Lambda\right)+\right. \\
& +b_{\left[j / N_{S}\right]}^{(v)} g^{(v)}\left(t-\lambda_{j, 0}-T_{d}^{(v)}-d_{\left[j / N_{S}\right]}^{(v)}-T_{d}^{(1)}-m \Lambda\right)+ \\
& +g^{(v)}\left(t+T_{f}-\lambda_{j, 0+1}-T_{d}^{(1)}-m \Lambda\right)+b_{\left[(j-1) / N_{S}\right]}^{(v)} \times \\
& \left.\left.\times g^{(v)}\left(t+T_{f}-\lambda_{j, 1}-T_{d}^{(v)}-d_{\left[(j-1) / N_{S}\right]}^{(v)} \Lambda-T_{d}^{(1)}-m \Lambda\right)\right)\right] \mathrm{d} t
\end{aligned}
$$

where $\lambda_{j, k}=\left(c_{j-k}^{(v)}-c_{j}^{(1)}\right) T_{c}+\tau_{v}$. Note that, due to the fact that $T_{m d s} \leq T_{d}^{(1)}$, all integrals where the integrand is the reference pulse times the interference pulse will be zero. Thus the term (22) can be rewritten as

$$
\begin{aligned}
& I_{m, 1, j, A}=\sum_{v=2}^{N_{u}} E_{p}\left[R_{1 v}\left(\lambda_{j, 0}+m \Lambda, m \Lambda\right)+b_{\left\lfloor j / N_{S}\right\rfloor}^{(v)} \times\right. \\
& \times R_{1 v}\left(\lambda_{j, 0}+T_{d}^{(v)}+d_{\left\lfloor j / N_{S}\right\rfloor}^{(v)} \Lambda+m \Lambda, m \Lambda\right)+ \\
& +R_{1 v}\left(\lambda_{j, 1}-T_{f}+m \Lambda, m \Lambda\right)+b_{\left\lfloor j-1 / N_{S}\right\rfloor}^{(v)} \times \\
& \left.\times R_{1 v}\left(\lambda_{j, 1}-T_{f}+T_{d}^{(v)}+d_{\left\lfloor j-1 / N_{S}\right\rfloor}^{(v)} \Lambda+m \Lambda, m \Lambda\right)\right]
\end{aligned}
$$

where

$$
R_{m n}(x, y)=\int_{y}^{y+T_{c o r r}} g^{(m)}(t) g^{(n)}(t-x) \mathrm{d} t
$$

Note that in (23) only the first and the fourth addend take a nonzero value for $m=0$, while for $m \neq 0$ all addends take the zero value. After averaging over all possible TH codes, symbols and $\tau_{v}$, the second order moment of $I_{m, 1, j, A}$ (only for $m=0$ ) can be obtained as

$$
\begin{aligned}
& E\left(I_{m=0,1, j, A}^{2}\right)=\frac{E_{p}^{2}}{T_{f}} \sum_{v=2}^{N_{u}}\left[\int_{-\infty}^{\infty} R_{1 v}^{2}(\tau, 0) \mathrm{d} \tau+\right. \\
& \left.+\frac{1}{(M / 2)} \sum_{d=0}^{(M / 2)} \int_{-\infty}^{\infty} R_{1 v}^{2}\left(\tau+T_{d}^{(v)}+d \Lambda-T_{f}, 0\right) \mathrm{d} \tau\right]
\end{aligned}
$$

The second order moment of $I_{m, 1, j, B}$ is derived as

$$
\begin{aligned}
& E\left(I_{m=0,1, j, B}^{2}\right)=\frac{E_{p}^{2}}{T_{f}} \sum_{v=2}^{N_{u}}\left[\int_{-\infty}^{\infty} R_{1 v}^{2}\left(\tau-T_{d}^{(1)}-m \Lambda, 0\right) \mathrm{d} \tau+\right. \\
& +\frac{1}{(M / 2)} \sum_{d=0}^{(M / 2)-1} \int_{-\infty}^{\infty} R_{1 v}^{2}\left(\tau+T_{d}^{(v)}+d \Lambda-T_{d}^{(1)}-m \Lambda, 0\right) \mathrm{d} \tau+ \\
& \left.+\frac{1}{(M / 2)} \sum_{d=0}^{(M / 2)-1} \int_{-\infty}^{\infty} R_{1 v}^{2}\left(\tau+T_{d}^{(v)}+d \Lambda-T_{d}^{(1)}-m \Lambda-T_{f}, 0\right) \mathrm{d} \tau\right]
\end{aligned}
$$

The contribution of MUI-times-noise term at the $m^{\text {th }}$ correlator output is

$$
\begin{aligned}
& I_{m, 2}=\int_{T_{1, m}}\left[n(t) i\left(t-T_{d}^{(1)}-m \Lambda\right)+n\left(t-T_{d}^{(1)}-m \Lambda\right) i(t)\right] \mathrm{d} t= \\
& =\sum_{j=0}^{N_{S}-1}\left[I_{m, 2, j, A}+I_{m, 2, j, B}\right]
\end{aligned}
$$

$I_{m, 2, j, A}$ can be written as

$$
\begin{aligned}
& I_{m, 2, j, A}=\sqrt{E_{p}} \sum_{v=2}^{N_{u}} \int_{T_{i, m}} n(t)\left[g^{(v)}\left(t-c_{j}^{(v)} T_{c}-\tau_{v}-T_{d}^{(1)}-m \Lambda\right)+\right. \\
& +b_{\left[j / N_{S}\right]}^{(v)} g^{(v)}\left(t-c_{j}^{(v)} T_{c}-T_{d}^{(v)}-d_{\left[j / N_{S}\right]}^{(v)} \Lambda-\tau_{v}-T_{d}^{(1)}-m \Lambda\right)+ \\
& +g^{(v)}\left(t+T_{f}-c_{j-1}^{(v)} T_{c}-\tau_{v}-T_{d}^{(1)}-m \Lambda\right)+b_{\left[(j-1) / N_{S}\right]}^{(v)} \times \\
& \left.\left.\times g^{(v)}\left(t+T_{f}-c_{j-1}^{(v)} T_{c}-T_{d}^{(v)}-d_{\left[(j-1) / N_{S}\right]}^{(v)} \Lambda-T_{d}^{(1)}-m \Lambda\right)\right)\right] \mathrm{d} t
\end{aligned}
$$

The second order moment of $I_{m, 2, j, A}$ is then 


$$
\begin{aligned}
& E\left(I_{m, 2, j, A}^{2}\right)=\frac{N_{o} E_{p}}{2 T_{f}} \sum_{v=2}^{N_{u}} \int_{-\infty}^{\infty}\left[\int_{0}^{T_{\text {corr }}}\left(g^{(v)}(t-\tau)\right) \mathrm{d} t+\right. \\
& \left.+\frac{1}{(M / 2)} \sum_{d=0}^{(M / 2)-1} \int_{0}^{T_{\text {corr }}}\left(g^{(v)}\left(t-\tau-T_{d}^{(v)}-d \Lambda+T_{f}\right)\right)^{2} \mathrm{~d} t\right] \mathrm{d} \tau
\end{aligned}
$$

In a similar way the second order moment of $I_{m, 2, j, B}$ is derived as

$$
\begin{aligned}
& E\left(I_{m, 2, j, B}^{2}\right)=\frac{N_{o} E_{p}}{2 T_{f}} \sum_{v=2}^{N_{u}} \int_{-\infty}^{\infty}\left[\int_{0}^{T_{\text {corr }}}\left(g^{(v)}\left(t-\tau+T_{d}^{(1)}+m \Lambda\right)\right)^{2} \mathrm{~d} t+\right. \\
& +\frac{1}{(M / 2)} \sum_{d=0}^{(M / 2)-1} \int_{-\infty}^{\infty}\left(g^{(v)}\left(t-\tau-T_{d}^{(v)}-d \Lambda+T_{d}^{(1)}+m \Lambda\right)\right)^{2} \mathrm{~d} t+ \\
& \left.+\frac{1}{(M / 2)} \sum_{d=0}^{(M / 2)-1} \int_{0}^{T_{\text {corr }}}\left(g^{(v)}\left(t+T_{f}-\tau-T_{d}^{(v)}-d \Lambda+T_{d}^{(1)}+m \Lambda\right)\right)^{2} \mathrm{~d} t\right]
\end{aligned}
$$

MUI-times-MUI term can be written as:

$$
I_{m, 3}=\int_{T_{1, m}}\left[i(t) i\left(t-T_{d}^{(1)}-m \Lambda\right)+i\left(t-T_{d}^{(1)}-m \Lambda\right) i(t)\right] \mathrm{d} t
$$

By using the techniques described above, the variance of $I_{m, 3}$ can be obtained as

$$
\begin{aligned}
& \operatorname{var}\left(I_{m, 3}\right) \approx \frac{4 N_{s} E_{p}^{2}}{T_{f}^{2}} \sum_{v=2}^{N_{u}} \sum_{\substack{v^{\prime}=2 \\
v^{\prime} \neq v}}^{N_{u}} \int_{-\infty}^{\infty} \int_{-\infty}^{\infty}\left(\int_{0}^{T_{\text {corr }}} g^{(v)}\left(t-\tau_{v}\right) g^{\left(v^{\prime}\right)}\left(t-\tau_{v^{\prime}}\right) \mathrm{d} t \times\right. \\
& \left.\times \int_{0}^{T_{\text {corr }}} g^{(v)}\left(x-\tau_{v}\right) g^{\left(v^{\prime}\right)}\left(x-\tau_{v^{\prime}}\right) \mathrm{d} x\right) \mathrm{d} \tau_{v} \mathrm{~d} \tau_{v^{\prime}} \text { for all } m
\end{aligned}
$$

As it is shown in Fig. 1, after demodulation the detector processes $M / 2$ correlator outputs $\left\{C_{m, z}, 0 \leq m \leq((M / 2)-1)\right\}$ and selects the correlator output with the largest absolute value $\left|C_{m, z}\right|$. Then the sign of this magnitude $C_{m, z}$ is used to decide which of the two possible amplitudes has been transmitted. Using the techniques described in [21], the probability of the correct decision at the receiver when the symbol with $d_{z}^{(1)}=0$ and $b_{z}^{(1)}=1$ is sent, is equal to

$$
P_{c}=\int_{0}^{\infty}\left[\prod_{m=1}^{(M / 2)-1} \frac{1}{\sqrt{2 \pi}} \int_{-C_{0, z} / \sqrt{\operatorname{var}_{\text {tot }, m}}}^{C_{0, z} / \sqrt{\operatorname{var}_{\text {tot }, m}}} \exp \left(-x^{2} / 2\right) \mathrm{d} x\right] p\left(C_{0, z}\right) \mathrm{d} C_{0, z}
$$

where $p\left(C_{0, z}\right)$ is the probability density function (PDF) of the first correlator output for the $z^{\text {th }}$ symbol and it is defined as

$$
p\left(C_{0, z}\right)=\frac{1}{\sqrt{2 \pi \operatorname{var}_{t o t, m=0}}} \exp \left[-\frac{\left(C_{0, z}-E\left(\varepsilon_{p}\right)\right)^{2}}{2 \operatorname{var}_{t o t, m=0}}\right]
$$

and $\operatorname{var}_{t o t, m}$ is the sum of all variances at the $m^{\text {th }}$ correlator. Finally, the probability of symbol error can be calculated as

$$
P_{M}=1-P_{c}
$$

\section{RESULTS AND DISCUSSION}

In this section, the numerical results obtained using the expressions from previous sections are presented. System performances are examined when two different channel models, CM1 and CM2 [10], are used. CM1 describes a line of sight (LOS) scenario when the distance between transmitter and receiver is less than $4 \mathrm{~m}$, while CM2 describes the non-line of sight (NLOS) scenario for the same distance between transmitter and receiver. According to [19], $T_{m d s}$ is about $32 \mathrm{~ns}$ for both channel models used in this examination. The assumption from $[13,19]$ is adopted, where the pulse width $T_{p}$ is chosen to be $0.167 \mathrm{~ns}$, in order to avoid IPI caused by channel distortion. Front-end filter bandwidth at the receiver, $W$, is set to $2 / T_{p}[13,20]$.

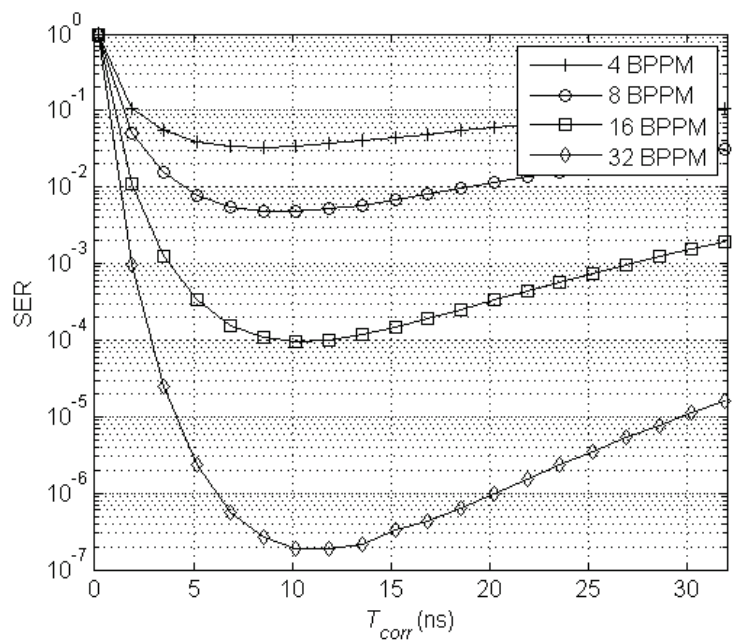

(a)

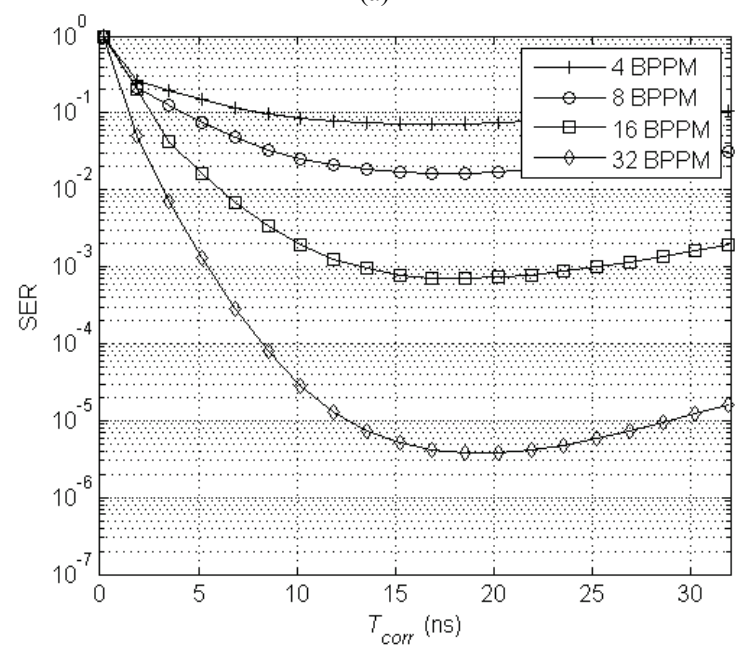

(b)

Figure 2 SER versus integration time interval duration, $T_{\text {corr }}$, for $M$-ary TR BPPM system, when $E_{b} / N_{0}=15 \mathrm{~dB}, N_{s}=1, N_{h}^{(1)}=10$ and $N_{u}=10$ (a) in CM1 (b) in CM2.

In Figs. 2 (a) and (b) the influence of the integration time interval duration, $T_{\text {corr }}$, on symbol error rate (SER) performances of $M$-ary TR BPPM system (for $M=4,8$, 16, 32) when CM1 and CM2 are used, is presented, respectively. Without loss of generality, for both channel models the value of $E_{b} / N_{0}$ is fixed to $15 \mathrm{~dB}$, where $E_{b}=E_{p} / \log _{2} M$ is bit energy. The first user is assumed to be a desired user, while the total number of users, $N_{u}$, is set to 10 . In $\mathrm{CM} 1$ the optimal integration time interval 
durations are found to be $8.5,10.2,10.3$ and $11.9 \mathrm{~ns}$ for $M$ $=4,8,16$ and 32 , respectively. When CM2 is used, these optimal integration time interval durations are 16.9, 17.0, 18.4 and 18.5 ns for $M=4,8,16$ and 32, respectively. In CM2 signal energy is dispersed on more multipath components than in CM1, due to NLOS scenario. The amplitudes of the components that arrive at the receiver are generally lower and additionally the components delay is higher in CM2. Thus, for all values of $M$ the optimal integration time interval duration required for achieving the best SER performances is higher in CM2.

On the other hand, with the increasing of integration time interval duration, the noise-time-noise component defined with (20) takes a higher value and thus the optimal integration time interval duration is obtained as a trade-off between the collected energy of desired signal and collected noise. When the integration time interval duration is larger than its optimal value, the amount of additionally collected noise becomes higher than the amount of desired signal in this part of time, and consequently SER performances decrease.

Figs. 3 (a) and (b) present SER performances of $M$ ary TR BPPM $(M=4,8,16,32)$ in a multi-user environment for optimal $T_{\text {corr }}$ obtained from Figs. 2(a) and 2(b), and minimal $T_{f}$ defined in (7), for CM1 and CM2, respectively.

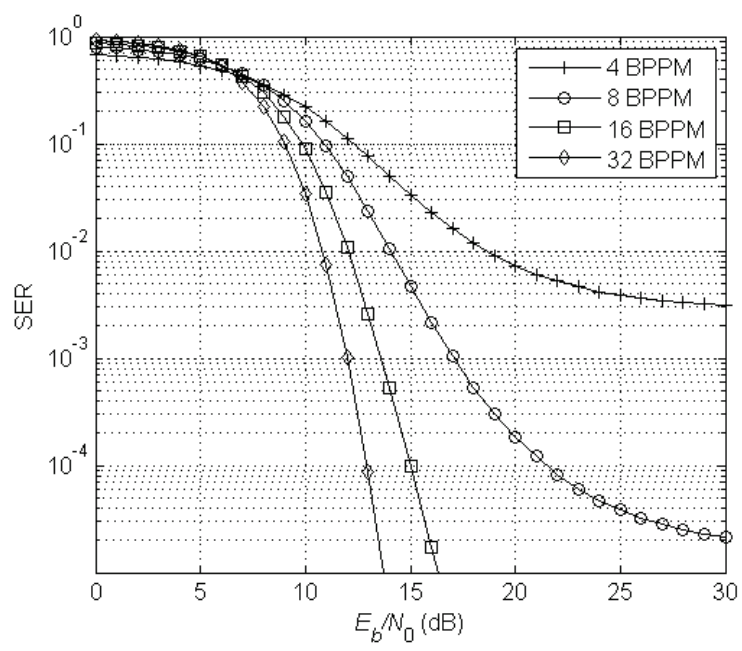

(a)

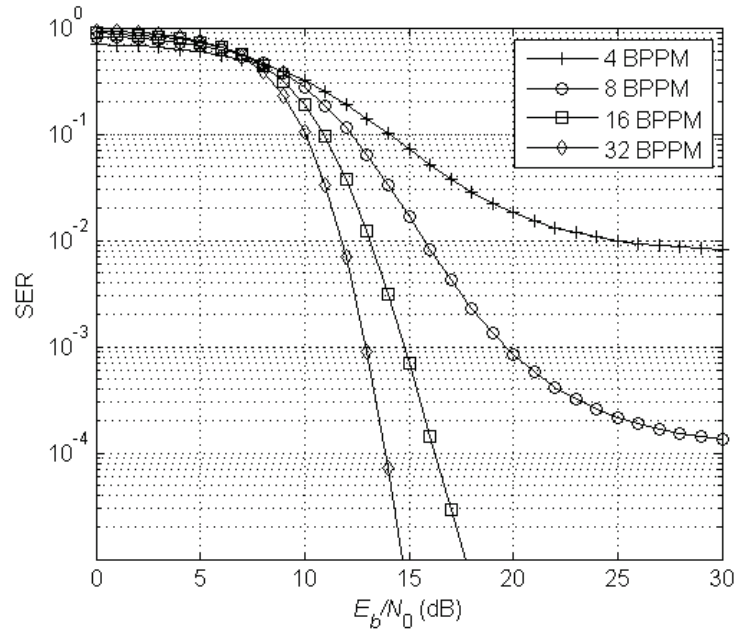

(b)

Figure 3 SER versus $E_{b} / N_{0}$ for different modulation levels, $M$, when $N_{s}=1$, $N_{h}^{(1)}=10$ and $N_{u}=10(\mathrm{a})$ in CM1 (b) in CM2
When modulation level increases, the SER performances significantly rise, what is the characteristic of the multi-level orthogonal modulation scheme. In order to collect the equal amount of desired signal energy in $\mathrm{CM} 2$ as in $\mathrm{CM} 1$, larger integration time interval duration is required in CM2 than in CM1 (due to larger channel delay spread). Thus for equal amount of collected desired signal energy, more noise is captured in CM2 and consequently SER performances are quite lower than in CM1.

Figure 4. shows how the number of frames, $N_{S}$, influences SER performances for different modulation levels $(M=4,8)$ in CM1. In general, when $N_{S}$ increases, SER performances rise. On the other hand, e.g. to achieve SER of $10^{-4}$ for $M=8$ when $N_{S}=4$, it is needed about 3 dB less $E_{b} / N_{0}$ than for $N_{S}=2$. This gain is at the cost of the data rate, since due to more frames per symbol the symbol duration is increased. Similar relation between $N_{S}$ and SER performances is obtained for $\mathrm{CM} 2$ and thus these results are not particularly presented.

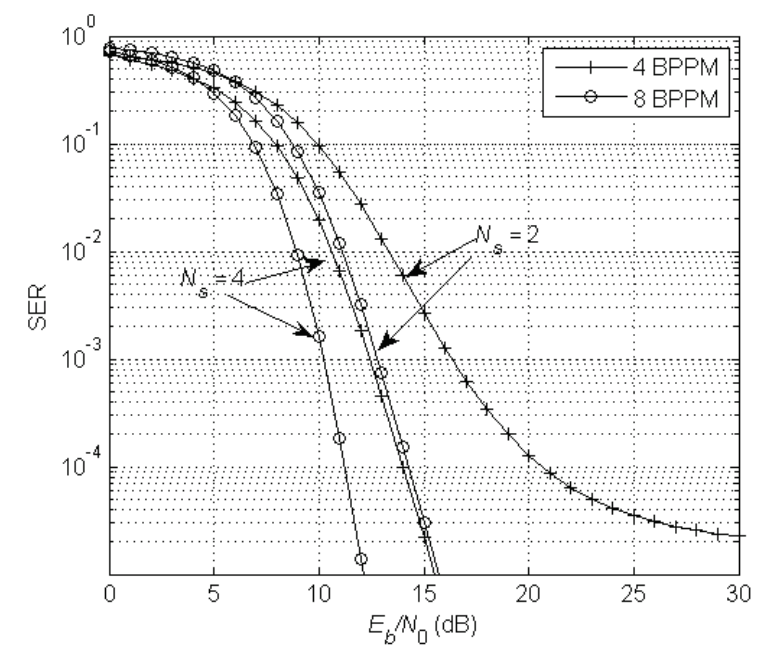

Figure 4 SER versus $E_{b} / N_{0}$ for different number of frames, $N_{s}$, when $N_{u}=10$ and $N_{h}^{(1)}=N_{u}$ in $\mathrm{CM} 1$

The performances of the proposed BPPM scheme are compared to these of the PPM scheme for the fixed bit rate and two different modulation levels. The results are presented in Fig. 5. It can be seen that the BPPM scheme slightly outperforms the PPM scheme in terms of SER for the whole considered range of $E_{b} / N_{0}$. This can be explained by the fact that the probability of the correct decision at the receiver is higher for the BPPM scheme than for the PPM scheme due to half a number of correlators. This leads to a lower possibility of missing the correct correlator output for the BPPM scheme. Consequently, the probability of the symbol error is lower for the BPPM scheme than for the PPM scheme.

The influence of the number of users on SER performances for the proposed BPPM scheme with different modulation levels is presented in Fig. 6. Clearly, as the number of users rises, the SER performances decrease for the same modulation level due to an increase in MUI

For the maximum considered number of users $\left(N_{u}=\right.$ 50), the achieved SER for $M=4,8,16$ and 32 is 0.18 , $0.12,0.08$ and 0.04 , respectively. On the other hand, the system complexity is increased when $M$ is higher. Thus, 
depending on required number of users, the trade-off between the SER performances and system complexity should be done.

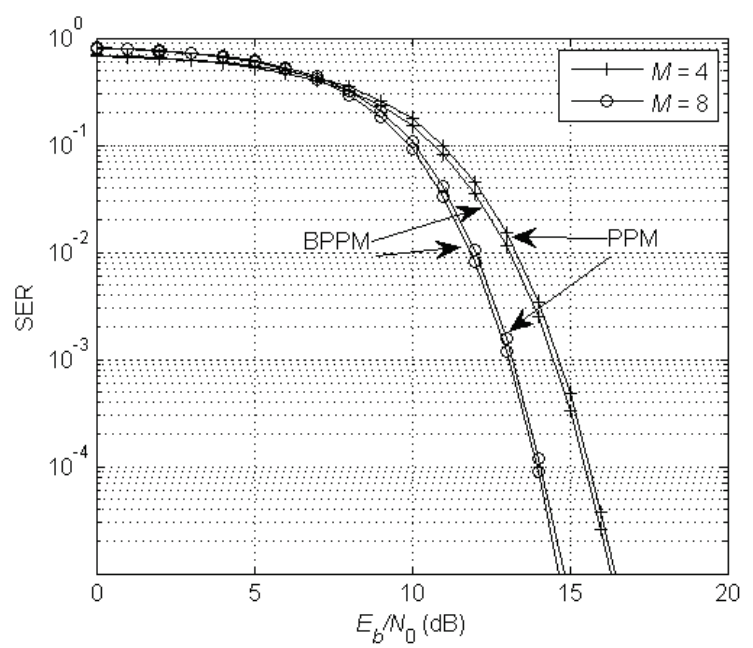

Figure 5 SER versus $E_{b} / N_{0}$ for fixed bit rate (1 Mbps) and different modulation levels for BPPM and PPM schemes in CM1, when $N_{s}=1, N_{u}=10$, $N_{h}^{(1)}=N_{u}$

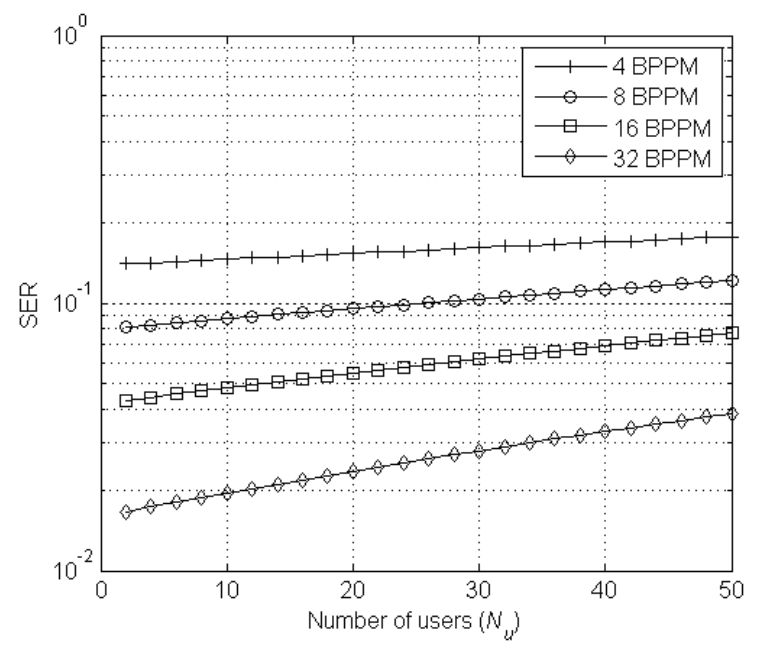

Figure 6 Influence of number of users, $N_{u}$, on SER performances for fixed bit rate $(1 \mathrm{Mbps})$ and $E_{b} / N_{0}=10 \mathrm{~dB}, N_{s}=1, N_{h}^{(1)}=N_{u}$, in CM1

\section{CONCLUSION}

In this paper the BPPM scheme for the application in TR IR-UWB systems is proposed. The SER performances of the proposed scheme have been analyzed over 805.15.3.a CM1 and CM2 in multi-user environment. It is shown that the BPPM scheme slightly outperforms the PPM scheme in terms of SER for the same modulation level, whereas the BPPM receiver hardware complexity is significantly reduced (i.e. the number of correlators in the $\mathrm{BPPM}$ receiver is half a number of correlators in the PPM receiver). Due to a lower number of modulation slots in the BPPM scheme, the symbol duration is shorter than in the PPM scheme and thus the achievable data rate for the same modulation level is higher for the BPPM scheme. These properties of the BPPM scheme make it very attractive for TR IR-UWB systems.

\section{Acknowledgements}

This work was supported by the Croatian Ministry of Education, Science and Sports through the projects No. 165-0361630-1636 and 165-0361630-3049.

\section{REFERENCES}

[1] Scholtz, R. A. (1993). Multiple access with time-hopping impulse modulation. IEEE Military Communications Conference, Boston, 447-450. https://doi.org/10.1109/MILCOM.1993.408628

[2] Le Marter, C. J. \& Giannakis, G. B. (2000). All-digital PAM impulse radio for multiple-access through frequencyselective multipath. IEEE Global Telecommunications Conference, San Francisco, 77-81. https://doi.org/10.1109/GLOCOM.2000.891694

[3] Win, M. Z. \& Scholtz, R. A. (2000). Ultra-wide bandwidth time-hopping spread-spectrum impulse radio for wireless multiple-access communications. IEEE Transactions on Communications, 48(4), 679-691. https://doi.org/10.1109/26.843135

[4] Shen, Y. S. et al. (2010). A High-Capacity TH MultipleAccess UWB System With Performance Analysis. IEEE Transactions on Vehicular Technology, 59(2), 742-753. https://doi.org/10.1109/TVT.2009.2035130

[5] Ghavami, M. et al. (2002). A Novel UWB Pulse Shape Modulation System. Wireless Personal Communications, 23(1), 105-120. https://doi.org/10.1023/A:1020953424161

[6] Wei, L. \& Gulliver, T. A. (2007). On-Off Keying UltraWideband Communication with Adaptive Overcomplete Dictionary Detection. Canadian Conference on Electrical and Computer Engineering, Vancouver, 1187-1190.

[7] Welborn, M. L. (2001). System considerations for ultrawideband wireless networks. Radio and Wireless Conference, Waltham, 5-8. https://doi.org/10.1109/RAWCON.2001.947480

[8] Zhao, B., Chen, Y., \& Green, R. (2012). Hard-Input-HardOutput Capacity Analysis of UWB BPSK Systems with Timing Errors. IEEE Transactions on Vehicular Technology, 61(4), 1741-1751. https://doi.org/10.1109/TVT.2012.2188418

[9] Herceg, M., Švedek, T., \& Matić, T. (2010). Pulse Interval Modulation for Ultra-High Speed IR-UWB Communications Systems. EURASIP Journal on Advances in Signal Processing, http://asp.eurasipjournals.com/ content/2010/1/658451(12.07.2015) https://doi.org/10.1155/2010/658451

[10] Molisch, A. F., Foerster, J. R., \& Pendergrass, M. (2003). Channel models for ultrawideband personal area networks. IEEE Wireless Communications, 10(6), 14-21. https://doi.org/10.1109/MWC.2003.1265848

[11] Shaban, H., \& El-Nasr, M. A. (2013). Amplify-andForward Cooperative Diversity for Green UWB-Based WBSNs. The Scientific World Journal, http://www.hindawi.com/journals/tswj/2013/785840/ (14.07.2015) https://doi.org/10.1155/2013/785840

[12] Hoctor, R. T. \& Tomlinson, H. W. (2002). An Overview of Delay-Hopped, Transmitted-Reference RF Communications. GE Research \& Development, Technical Information Series.

[13] Kim, D. I. \& Jia, T. (2008). M-ary orthogonal coded/balanced ultra-wideband transmitted-reference systems in multipath. IEEE Transactions on Communications, 56(1), 102-111. https://doi.org/10.1109/TCOMM.2008.050310

[14] Jia, T. \& Kim, D. I. (2008). Multiple Access Performance of Balanced UWB Transmitted-Reference Systems in 
Multipath. IEEE Transactions on Wireless Communications, 7(3), 1084-1094. https://doi.org/10.1109/TWC.2008.060864

[15] Chao, Y. L. \& Scholtz, R. A. (2004). Multiple Access Performance of Ultra-Wideband Transmitted Reference Systems in Multipath Environments. IEEE Wireless Communications and Networking Conference, Atlanta, 1788-1793.

[16] Xu, Z. \& Sadler, B. M. (2006). Multiuser Transmitted Reference Ultra-Wideband Communication Systems. IEEE Journal on Selected Areas in Communications, 24(4), 766772. https://doi.org/10.1109/JSAC.2005.863826

[17] Li, L. \& Townsend, J. K. (2010). M-ary PPM for Transmitted Reference Ultra-Wideband Communications. IEEE Transactions on Communications, 58(7), 19121917. https://doi.org/10.1109/TCOMM.2010.07.080675

[18] Zhang, H. \& Gulliver, T. A. (2005). Biorthogonal Pulse Position Modulation for Time-Hopping Multiple-Access UWB Communications. IEEE Transactions on Wireless Communications, 4(3), 1154-1162. https://doi.org/10.1109/TWC.2005.846969

[19] Jia, T. \& Kim, D. I. (2007). Analysis of Channel-Averaged SINR for Indoor UWB Rake and Transmitted Reference Systems. IEEE Transactions on Communications, 55(10), 2022-2032. https://doi.org/10.1109/TCOMM.2007.906435

[20] Kim, D. I. (2009). Multiuser performance of M-ary orthogonal coded/balanced UWB transmitted-reference systems. IEEE Transactions on Communications, 57(4), 1013-1024. https://doi.org/10.1109/TCOMM.2009.04.070146

[21] Proakis, J. G. (2001). Digital Communications. $4^{\text {th }}$ ed. New York, McGraw-Hill.

\section{Contact information:}

Mario VRANJEŠ, PhD., Assistant Professor

J. J. Strossmayer University of Osijek

Faculty of Electrical Engineering, Computer Science and Information

Technology

Kneza Trpimira 2B, 31000 Osijek, Croatia

+385 31 224670, mario.vranjes@etfos.hr

Denis VRANJEŠ, PhD., Senior Instructor

J. J. Strossmayer University of Osijek

Faculty of Electrical Engineering, Computer Science and Information

Technology

Kneza Trpimira 2B, 31000 Osijek, Croatia

+385 31 224760, denis.vranjes@etfos.hr

Tomislav MATIĆ, PhD., Associate Professor

J. J. Strossmayer University of Osijek

Faculty of Electrical Engineering, Computer Science and Information

Technology

Kneza Trpimira 2B, 31000 Osijek, Croatia

+385 31 224732, tomislav.matic@etfos.hr 